\title{
Primeras evidencias sobre el Paleoindio en Bolivia
}

JoRGE ARELlano

\section{Introducción}

La presente comunicación trata de las primeras investigaciones de sitios paleoindios realizadas por el Instituto Nacional de Arqueología de Bolivia, en la región de Lípez, departamento de Potosí, y que contaron con la participación de Eduardo Berberian, director del Instituto de Antropología de la Universidad de Córdoba, Argentina.

Estas investigaciones constituyen, en el momento, un paso en el desarrollo del conocimiento científico del Paleoindio en Bolivia, las cuales, sin duda alguna, en el futuro alcanzarán mayor relieve.

En este sentido, la región de Lípez se presenta como una de las más aptas y apropiadas para una preservación de restos arqueológicos, que, a su vez, están relacionados con un ambiente pleistocénico.

Los estudios sobre esta región son muy limitados, razón por la cual la bibliografía arqueológica es escasa. De ésta podemos mencionar, en primer lugar, las observaciones efectuadas por Courty, miembro de la misión científica francesa junto a Créqui-Monfort y Senechal de la Grange (1903-1904), en los cerros Relave y Huanco, donde señala la presencia de instrumentos líticos, contemporáneos, según él, con el Paleolítico europeo (Courty 1913: 43).

Más tarde, en 1958, una misión de la Universidad de Cambridge (Inglaterra) realizó investigaciones geomorfológicas y arqueológicas al este de la provincia de Antofagasta (Chile), abarcando una parte del altiplano boliviano, como se menciona en el trabajo publicado por Barfield (1961), el cual da algunos datos sobre sitios arqueológicos en el sector de laguna Colorada. Le Paige (1964) también proporciona algunos datos de sitios arqueológicos ubicados en Lípez, relacionando artefactos líticos de la región con otros del norte chileno.

Finalmente se tiene el trabajo de Berberian y Arellano (1978), sobre el cual está basada parte de la presente comunicación, puesto que es, por el momento, el único aporte al conocimiento científico del Paleoindio en Bolivia. Sin embargo, debemos también dejar claro que existe al respecto una serie de modificaciones, especialmente en lo que se refiere a la denominación de los artefactos, ya que consideramos que existe una nueva visión tipológica de ellos, de acuerdo a las conclusiones adoptadas en el seminario efectuado en Antofagasta durante el mes de octubre de 1978.

\section{El medio ambiente actual}

La región investigada se encuentra limitada a la parte sur del altiplano o Puna, y comprende, en la actualidad, a las provincias Nor y Sur Lípez del departamento de Potosí (Figuras 1 y 2). Estas dos provincias, de acuerdo a la memoria explicativa del mapa ecológico de Bolivia, poseen dos regiones definidas: la templada de tierras altas y la templada andina, cada una con una serie de subdivisiones en pisos y zonas de vida, presentando, sin embargo, en algunos aspectos, similares características, como ser: alta incidencia de radiación solar durante el día y alta incidencia de radiación nocturna, que da lugar a cambios bruscos de temperatura, por lo que también la región carece de importancia agrícola, salvo en escasos lugares donde se cultivan quinoa, cebada y papa.

Ahlfeld (1973) considera como Puna el paisaje natural de esta porción del país, con dos regiones morfológicas: la primera, que comprende la parte occidental y que contiene los volcanes y bolsones sin desagüe, con sus lagos y salares, y la segunda, la oriental, donde se ubica la cordillera de Lípez, que constituye el último ramal de la cordillera Central. Para Muñoz Reyes (1977), Lípez entra en la categoría del altiplano intermontano, y se caracteriza por la existencia de una serie de cuencas menores y bolsones fisiográficos, en los que se han formado pequeños lagos o lagunas, amargas y saladas.

En un estudio geomorfológico de la región (Fernández 1972: 70) se considera que Lípez: 


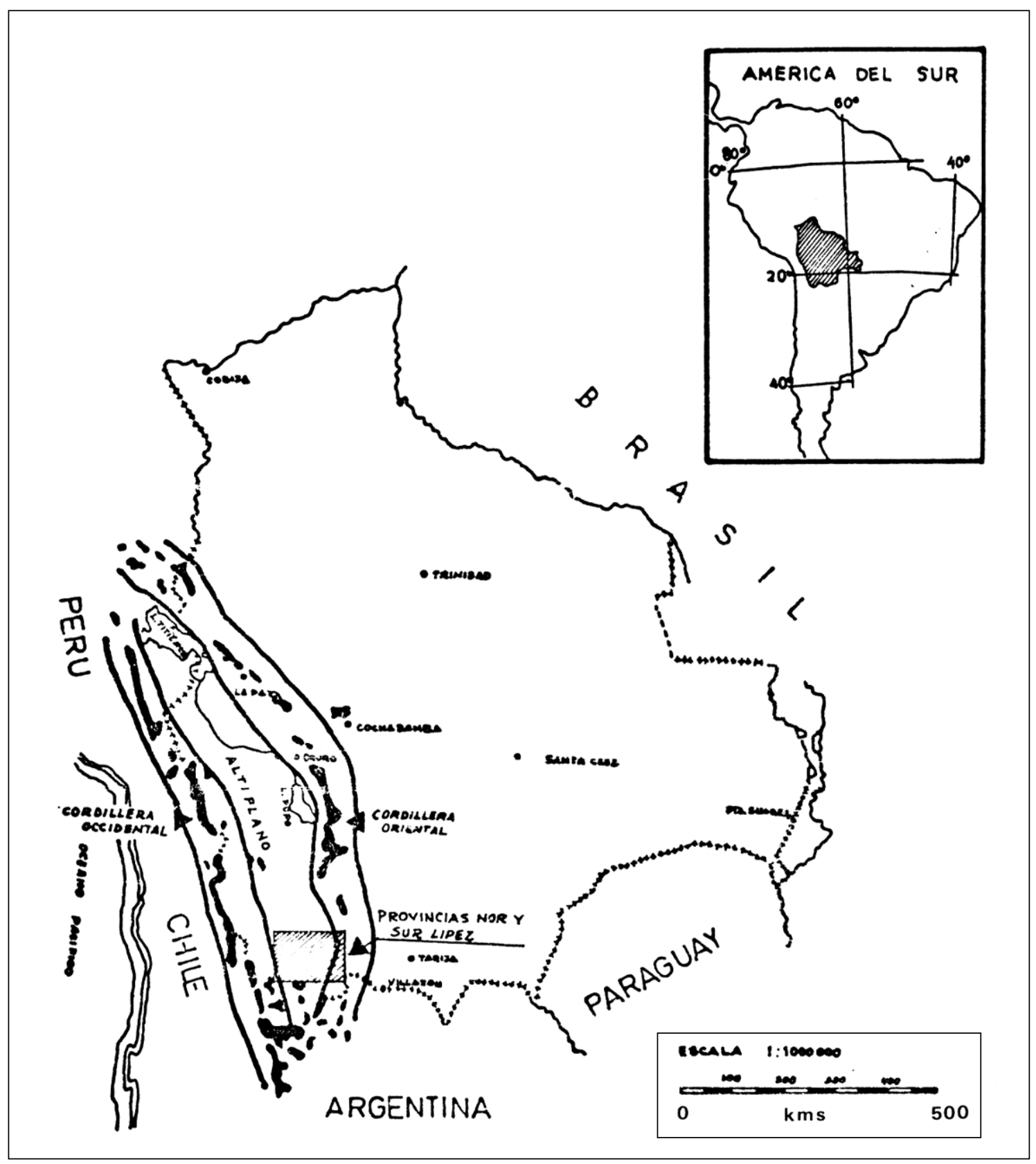

Figura 1. Mapa de ubicación del área de estudio.

"morfoestructuralmente forma parte de tres unidades bien definidas, que de oeste a este son: cordillera Occidental o de los volcanes, cuenca altiplánica parte sur y bloque paleozoico andino, que se trata de la cordillera de los Lípez".

Quizá esta última apreciación de la zona es la que nos da una idea cabal de su morfología y nos ayuda a definir su clima y sus variedades. De acuerdo entonces a lo mencionado se tienen tres tipos de clima: los ramales de la cordillera Oriental y Occidental pertenecen al clima polar de la alta montaña (EB en la clasificación de Köeppen). En la cuenca altiplánica, situada entre los dos cordones montañosos, se tienen dos tipos de clima: el de tundra (ET de la nomenclatura de Köeppen) y el de estepa, con invierno seco y frío (BSwk según Köeppen). Los ríos que drenan las provincias Nor y Sur Lípez corresponden al sistema central o lacustre 


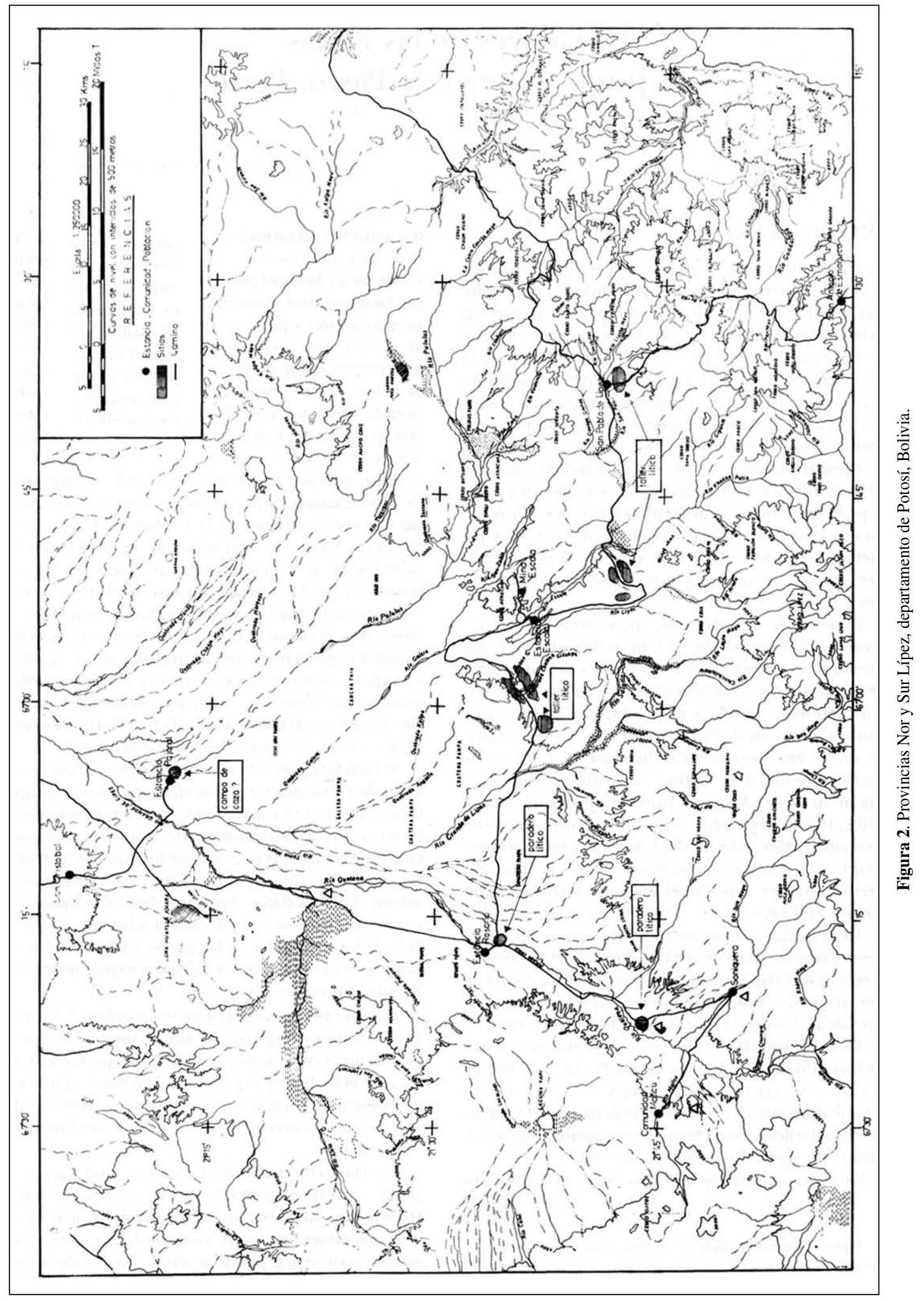


interior, de carácter mediterráneo. En este sentido, los ríos Grande de Lípez y Quetena son los que recogen todas las aguas de las demás corrientes fluviales, que atraviesan la extensa planicie producto de los lagos pleistocénicos, para desembocar luego en el salar de Uyuni. El Puka Mayu es otro río de importancia, que también lleva sus aguas al salar de Uyuni, y que en cierta parte corre en forma paralela al Grande de Lípez. Hacia el sur, existen muchos salares, borateras y lagunas amargas, residuos, todos ellos, de lagos pleistocénicos formados por el escurrimiento de las aguas de los volcanes vecinos. Altos topográficos notables en la región de Lípez lo constituyen los volcanes situados en la cordillera Occidental, entre los cuales podemos mencionar por su importancia a los siguientes: volcán Ollagüe $(5830 \mathrm{~m})$, volcán San Pablo (5810 m), cerro Ascotán (5480 m), volcán Apagado (5680 m), volcán Linzor (5660 m), volcán Jorgencal $(5540 \mathrm{~m})$, volcán Putana $(5690 \mathrm{~m})$, cerro Sairecabur (5970 m), cerro Licancabur $(5930 \mathrm{~m})$ y volcán Suriques $(5.710 \mathrm{~m})$. Mientras en la cordillera de Lípez se distinguen las siguientes cumbres: cerro Lípez (5.930 m), cerro Santa Isabel (5903 m), cerro Bonete $(5630 \mathrm{~m})$, cerro Nuevo Mundo $(6020 \mathrm{~m})$, cerro Bravo $(5740 \mathrm{~m})$ y cerro Zapaleri $(5650 \mathrm{~m})$. Dentro de este ambiente extremadamente árido se encuentran algunas microáreas protegidas, donde se han podido desarrollar poblaciones mediante una subsistencia basada en una agricultura reducida, complementada con la crianza de especies animales como llamas, alpacas y ovejas. Algunas de las poblaciones actuales se ubican muy cercanas a las cabeceras de los valles formados por los ríos Quetena y Lípez, y de otros que desaguan en éstos. De manera similar, los asentamientos arqueológicos tardíos se encuentran siempre relacionados con cursos de agua y en las faldas de las serranías. Por el contrario, los posibles yacimientos paleoindios se ubican en los diferentes niveles de terrazas que formaron los ríos y lagunas, donde se encuentran muchos de los artefactos desechados en sus actividades cinegenéticas y en "oquedades y nichos abiertos por deflación en afloramientos de lavas e ignimbritas, sobre todo en la región de cerro Bonete y cerro Lípez" (Fernández 1972: 73).

\section{El medio ambiente pleistocénico}

El medio ambiente pleistocénico y holocénico en el sur del altiplano fue muy particular y de características completamente dispares al ambiente del altiplano norte y, por supuesto, al de las altas montañas. Por tanto, debemos tener en cuenta que los primeros grupos humanos han debido desarrollar para cada uno de los medio ambientes un cierto tipo de habilidades de supervivencia, relacionándola posiblemente con la fauna existente. La misma que a su vez, seguramente, estaba mejor condicionada para una expansión horizontal en el amplio marco geográfico que constituyen los Andes.

De manera que consideramos de importancia esbozar un cuadro generalizado del Pleistoceno para poder luego dar ideas más o menos exactas de los eventos acaecidos en la parte altiplánica que nos ocupa.

Para los Andes altos se han podido observar cuatro ciclos de glaciación a base de depósitos glaciales y de gravas fluvioglaciales, "los que cubren una vasta extensión del borde occidental de la cordillera Oriental y el altiplano oriental" (Schlatter y Nerderlof 1966: 6). No obstante, donde se ha realizado un estudio mucho más profundo sobre glaciación en los Andes es en la cuenca de La Paz, cuenca que está relacionada en forma directa con la glaciación de montaña. Al respecto, Dobrovolny (1962) distingue cuatro etapas glaciales con otros tantos interglaciales, las cuales pueden ser adaptadas para los eventos pleistocénicos ocurridos en la cordillera Oriental de los Andes. De estas etapas glaciales, las tres primeras fueron los avances más grandes de los hielos en el sector norte de la cordillera Oriental. Se trató de glaciales pedemontanos, los que formaron grandes depósitos de till, que más tarde fueron erosionados y cubiertos por depósitos fluviales en cada una de las etapas interglaciales.

La última etapa glacial, es decir, la cuarta, a nuestro juicio la más importante, se refiere a glaciares de valle que "no fueron tan grandes como los de etapas anteriores y que ocuparon los valles de corrientes ya existentes" (Dobrovolny 1962: 82). En esta cuarta etapa glacial hubo "por lo menos dos avances grandes de los glaciares de valle con una subetapa interglacial" y una siguiente subetapa como reciente, donde se produjo la erosión y ensanchamiento de valles en forma intensa, acompañada por una serie de cambios climáticos, los cuales se los puede observar en la alteración que presentan los depósitos sedimentarios. Esta última apreciación se ve confirmada por investigaciones realizadas en el área de Challapata, donde se determinaron dos avances de glaciares separados por un "período de retroceso y de erosión" (Servant 1977: 25) y por estudios palinológicos efectuados por Graff (1977), de turberas postglaciales ubicadas en la cordillera Real y cordillera de Tres Cruces, de 
las que se obtuvieron resultados relacionados con el último período glacial y que por su importancia se las reproduce in extenso:

“- Durante la última época interglacial, el clima fue más húmedo y la temperatura unos $3^{\circ} \mathrm{C}$ más alta que la actual.

- En el último pleniglacial, el clima fue seco y la temperatura unos $6^{\circ} \mathrm{C}$ más baja que la actual.

- En un interestadial, el clima fue húmedo y la temperatura $1-2^{\circ} \mathrm{C}$ más alta que la actual.

- En la fase tardía de la última época glacial, el clima fue húmedo y la temperatura unos $4^{\circ} \mathrm{C}$ más baja que la actual, lo que causó la extensión máxima de los ventisqueros en la última época glacial.

- Entre aproximadamente 10000 y 8200 AP, se tuvo un período de calentamiento lento, con un clima relativamente seco.

- Entre 8200 y 3600 AP, se presentó el período más húmedo y cálido del postglacial, especialmente entre 5600 y 5200 AP, con temperaturas de unos $2^{\circ} \mathrm{C}$ más altas que las actuales.

- A partir de 3600 AP existió un período generalmente seco y más frío, apareciendo poco a poco la estepa andina."

De estas consideraciones, las tres últimas nos parecen de mayor importancia, puesto que muestran una idea de las condiciones ambientales paralelamente a una cronología.

Esta serie de elementos de juicio nos sirven para analizar de una forma más real los eventos pleistocénicos en el altiplano sur, donde la actividad volcánica tuvo un rol importante y que con seguridad ha subsistido hasta tiempos holocénicos, como menciona Avila (1978: 32), cuando indica que "la actividad volcánica principia en el suroeste boliviano en el Oligoceno, alcanzando una mayor difusión en el Mioceno, hasta llegar al paroxismo en el Holoceno". Estos volcanes pleistocénicos se encuentran muy bien conservados, y generalmente se pueden observar en ellos sus cráteres y coladas de lava.

Sin embargo, por el momento es imposible poder correlacionar los eventos volcánicos con la presencia de dos grandes lagos del Pleistoceno Tardío, que se ubicaron en la parte media y sur del altiplano y de los cuales sus remanentes son el lago Poopó, los salares de Coipasa, Uyuni, Empexa, Ascotán, etc., y toda la planicie salobre por la que corren actualmente los ríos Quetena, Grande de Lípez y Puka Mayu.

El lago pleistocénico de data más temprana, denominado Minchin, tenía aproximadamente una longitud de norte a sur de $400 \mathrm{~km}$ y un ancho máximo de $200 \mathrm{~km}$ (Figura 3). Este lago poseía una serie de islas y penínsulas, entre las que podemos mencionar por su importancia con la región estudiada a las de San Cristóbal, San Agustín, Colcha y la península formada por la serranía intersalar, que separa los salares Uyuni y Coipasa. Las terrazas de este lago muestran una serie de niveles de 40 a $80 \mathrm{~m}$, por encima del salar de Uyuni en su cota más baja (3.660 m.snm) y están cubiertos por una gran cantidad de "arrecifes estrematolíticos" (Servant 1977: 26).

Un segundo lago, o mejor dicho una segunda extensión lacustre, en la región lo constituye el denominado Tauca (Servant 1977: 26), con resabios de $60 \mathrm{~m}$ de altura sobre el nivel actual de los salares. Según Servant (1977), este segundo espejo lacustre habría tenido $43000 \mathrm{~km}^{2}$, y sería de data más temprana. La disminución de afluentes con agua corriente y la constante evaporación, producida por un clima presumiblemente muy seco, dio lugar a una reducción de las aguas con la consecuente concentración de sales, que posteriormente se depositaron para formar los grandes salares. También sobre este particular se postula un posible desagüe de carácter transitorio a través "de los salares de Chiguana-Ollagüe, Carcote y Ascotán, que habrían formado un canal en la época de su mayor expansión y que atravesaba la cordillera Occidental en la puerta de Ollagüe" (Ahlfeld y Branisa 1960: 162), canal que más tarde fue obstruido por depósitos volcánicos producto de erupciones, depósitos que actualmente forman las barreras del suroeste del salar de Ascotán. En cuanto a una ubicación cronológica de los lagos, no se tiene sino por el momento una datación para la última extensión lacustre, el denominado lago Tauca, con una datación por $\mathrm{C}^{14}$ que lo sitúa entre los 13000 y 10000 AP, coincidiendo con un importante retroceso de los glaciares (Servant 1977: 26). Mientras que para el precedente lago Minchin podemos sólo inferir su ubicación cronológica en la última época interglacial. Este fondo de altiplanicie, donde se ubicaron los lagos pleistocénicos, gradualmente se va elevando con dirección suroeste para pasar a 


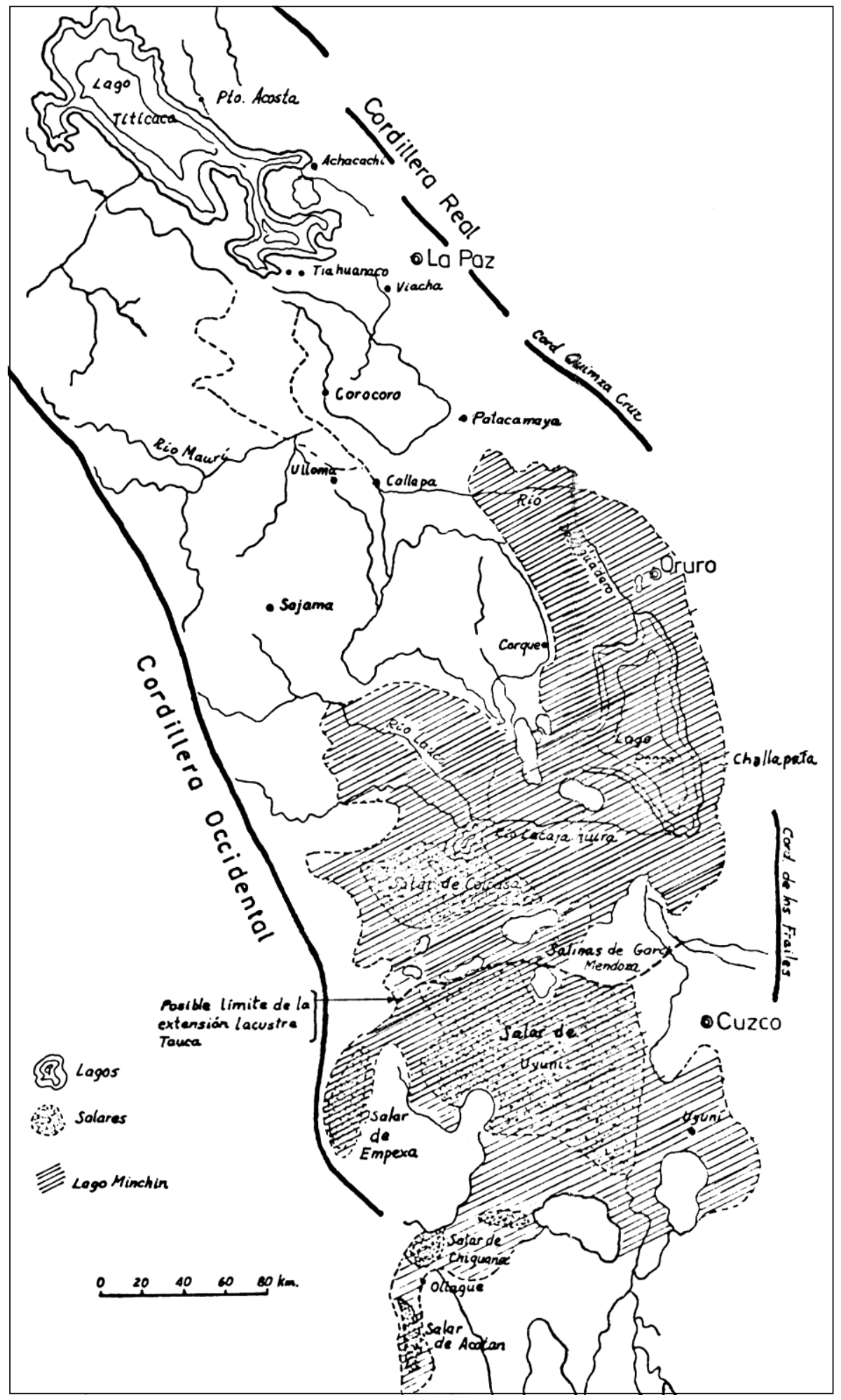

Figura 3. Croquis del lago pleistocénico Minchín y de la extensión lacustre Tauca. 
formar una serie de bolsones cerrados que al sur de Lípez llegan a tener una altura de 5000 m.snm. En este paisaje existen una serie de cuencas cerradas rodeadas por volcanes, en las que generalmente se encuentran lagunas salobres. En tanto que, hacia el sureste el río Lípez ha formado cañadones profundos, durante los ciclos de erosión posteriores a las etapas glaciales. En resumen, podemos considerar que el medio ambiente en el que se desenvolvieron los grupos humanos en este determinado espacio de tiempo, por lo menos entre 13000 y 3600 años AP, fue bastante óptimo en cuanto a temperatura, la que posiblemente se vio complementada con un calentamiento un poco más allá de lo normal, por las constantes erupciones volcánicas, suponiendo que por estos factores este medio ambiente nunca ha estado estable, lo que dio lugar a una constante migración de la fauna. En cuanto a la existencia de una flora y fauna particular, por las evidencias de restos de algas calcáreas y de calizas arenosas con diatomeas que necesitan para su desarrollo un ambiente lacustre de aguas límpidas, templadas y

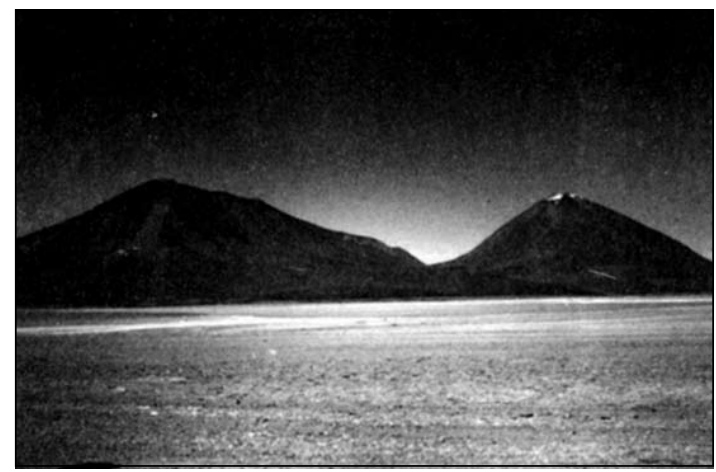

Figura 4. Vista de Laguna Verde con los volcanes Zoniquera y Licancabur, que forman la cordillera Occidental andina.

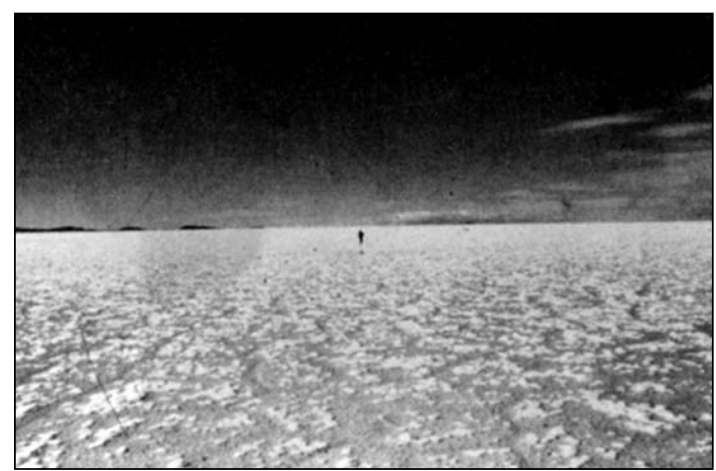

Figura 5. Una vista panorámica del salar de Uyuni, remanente del lago pleistocénico Minchín. de salinidad moderada, consideramos que la región ha estado poblada por una diversidad de especies vegetales, de las cuales en el presente sólo quedan algunas que se adaptaron a la extrema aridez (Figuras $4,5,6$ y 7 ). De la misma forma, la fauna habría sido muy variada, ya que aún la zona cuenta con especies animales silvestres de uso doméstico. Finalmente, no debemos dejar de lado la existencia de eventos tectónicos ocurridos durante el Pleistoceno, eventos que crearon cuencas cerradas con ambientes mucho más aptos para la supervivencia de una megafauna, como son las cuencas de Tarija y Padcaya, y a las cuales pudieron haber migrado grupos humanos.

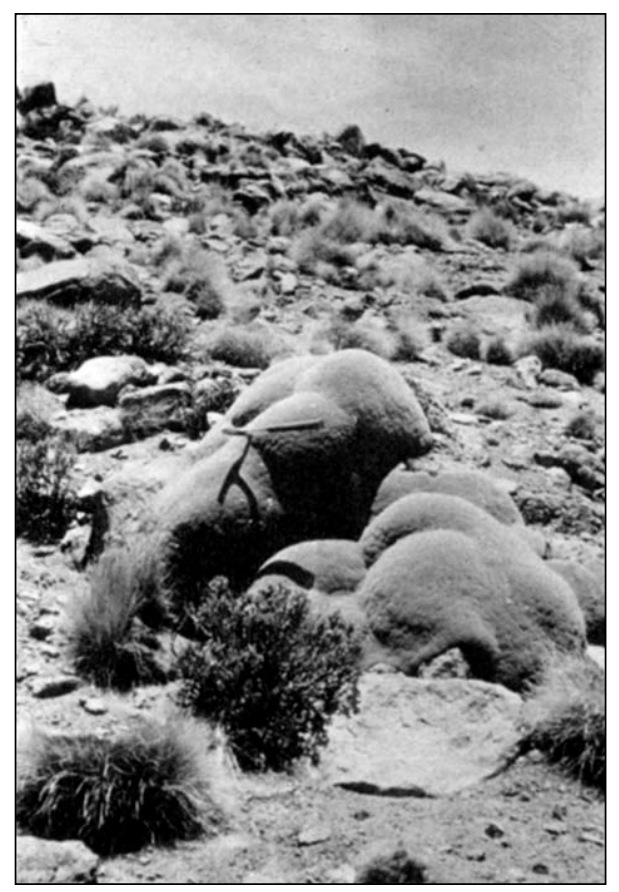

Figura 6. Vegetación característica de la zona; en primer plano, yareta.

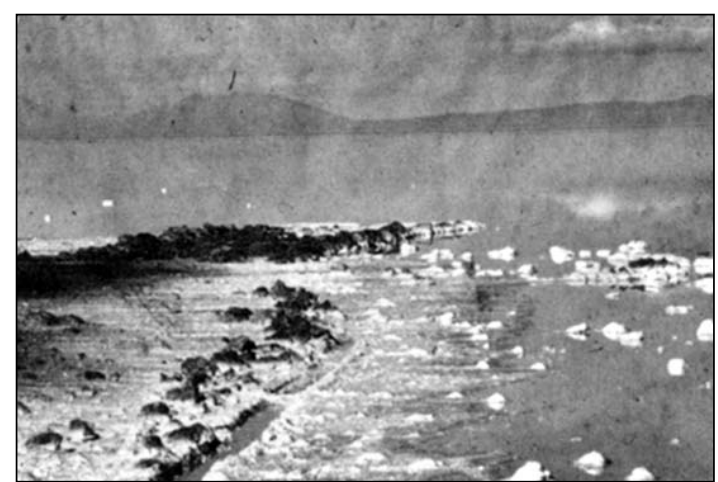

Figura 7. El salar de Uyuni durante la época de lluvias. 


\section{Los sitios}

Los sitios ubicados en el transcurso de las investigaciones realizadas en Lípez se los puede diferenciar en tres categorías: talleres líticos, paraderos y campos de caza, esta última con cierta reserva. La ubicación de cada uno de los sitios definidos en las anteriores categorías da una idea si no exacta por lo menos muy aproximada, de un medio ambiente al que se enfrentaban los grupos humanos que habitaron la región. Como se podrá apreciar, los talleres líticos se encuentran situados preferentemente sobre las terrazas superiores de los principales ríos que drenan la amplia planicie formada por el antiguo lago Minchin; estas terrazas ocurren cerca de las nacientes de cada una de las corrientes pluviales, es decir, cerca de las cadenas montañosas que circundan la región. Mientras que los paraderos y campos de caza están ubicados en el interior de la planicie. De manera similar, los posibles sitios de habitación (cuevas, abrigos y reparos) están situados en las serranías que presentan afloramientos rocosos y en un nivel mucho más alto, inclusive, al de las terrazas superiores de los ríos.

Algunos de estos abrigos y paredes rocosas presentan series de pinturas rupestres, en las que se observan figuras antropomorfas, zoomorfas y geométricas, aisladas y relacionadas entre sí. Aunque en principio, por los restos superficiales de fragmentos de cerámica que están diseminados en los alrededores se los considera correspondientes a etapas agroalfareras, no se puede descartar la posibilidad de que puedan tener una relación con grupos humanos del Paleoindio, o bien, con grupos de cazadores recolectores tempranos. Esto porque algunos de los abrigos muestran en cortes accidentales de la superficie una estratigrafía arqueológica bastante espesa.

Pasando a una descripción de los sitios, iniciaremos ésta con la de los talleres líticos, continuando posteriormente con los paraderos líticos para concluir con los campos de caza. Uno de los talleres líticos más importantes de la región es el que se ubica a una distancia de $3 \mathrm{~km}$ al sur de la mina Abaroa, sobre una serie de terrazas construidas por la acción erosiva y acumulativa del río Grande de Lípez, las que tienen una altura aproximada de $30 \mathrm{~m}$, sobre el lecho actual del río y una altura de $4000 \mathrm{~m}$.snm. El taller lítico contiene principalmente una industria de bifaces, distribuidas superficialmente de manera regular a ambos lados del camino, que desde mina Abaroa conduce a estancia Galena y mina Escala, en una extensión superior a los $10 \mathrm{~km}$ de longitud. Los artefactos bifaciales de los talleres líticos abaroenses tienen como características más importantes las siguientes: "formas amigdaloides, lanceoladas y rectangulares. Secciones lenticulares, biconvexas, planoconvexas y subtriangulares" (Berberian y Arellano 1978: 9). La talla ha sido efectuada por percusión y eventualmente presentan retoques. Muchos de los artefactos aún conservan parte de la superficie original del núcleo de la materia prima, en la que se observan pátina y rugosidades causadas por la acción eólica. Existen también artefactos unifaciales en escala limitada, los que tienen como características "formas lanceoladas, secciones planoconvexas y triangulares" (Berberian y Arellano 1978: 9); la talla fue por percusión y algunos presentan una carena central. Se puede también mencionar la existencia de raspadores (discoidales y subdiscoidales), raederas (triangulares y lanceoladas), algunos chopping-tools, choppers y una infinidad de lascas primarias y secundarias. La materia prima utilizada en este taller lítico fue una sola, obsidiana verdosa devitrificada (Figuras 8 y 12). Otro de los talleres líticos es el que se encuentra en la población de San Pablo de Sud Lípez, sobre unas terrazas vecinas a la mencionada población, las que aproximadamente tienen unos $40 \mathrm{~m}$ de altura. Los artefactos son mucho más desarrollados que los de los talleres abaroenses; se trata de bifaces, unifaces y de formas cercanas a puntas de proyectil, pero que en realidad son formas frustradas. Las bifaces y unifaces son mucho más pequeñas que las del Abaroense, tienen formas lanceoladas y fueron elaboradas por percusión. Las puntas de proyectil son unifaciales y bifaciales de formas lanceoladas, que indiscutiblemente derivan de los bifaces y unifaces mencionadas en primer término. Sus secciones son lenticulares, biconvexas en las bifaciales y planoconvexas en las unifaciales. Su elaboración fue realizada hasta ese punto por percusión antes de ser desechada (Figuras 9, 10 y 11).

En este taller lítico, complementando los artefactos indicados, se observan raederas, perforadores y gran cantidad de lascas. Para la elaboración de los artefactos se usaron dos clases de materia prima: basalto negro y obsidiana devitrificada de color verde olivo. En esta categoría de taller lítico también podemos incluir al sitio de río Quetena IV, donde se observan "instrumentos con características morfológicas similares" al de San Pablo de Sud Lípez "pero trabajadas en ópalo" (Berberian y Arellano 1978: 10). Consideramos como "paraderos" a aquellos talleres líticos de escasa extensión y que se encuentran predominantemente 


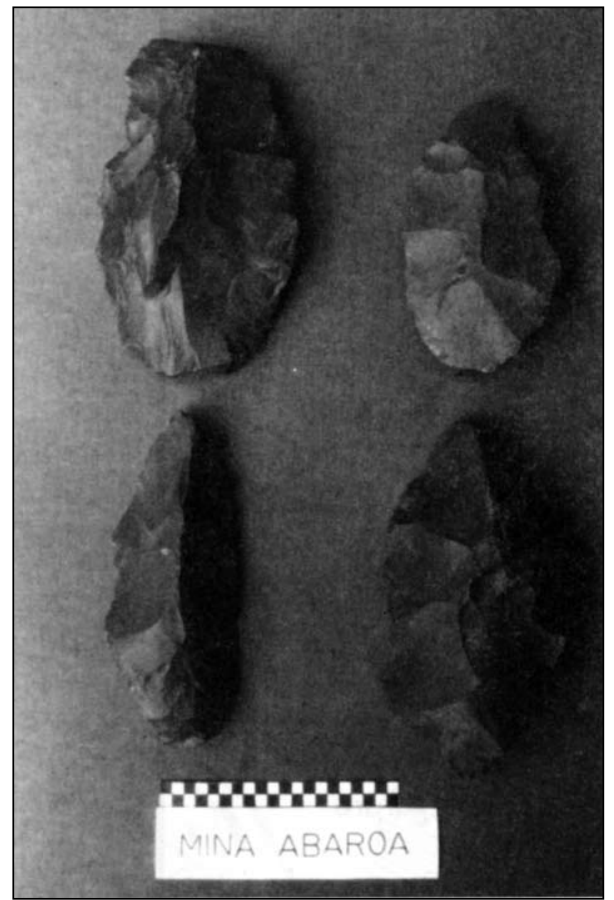

Figura 8. Artefactos líticos de mina Abaroa, elaborados en obsidiana devitrificada de color verde.

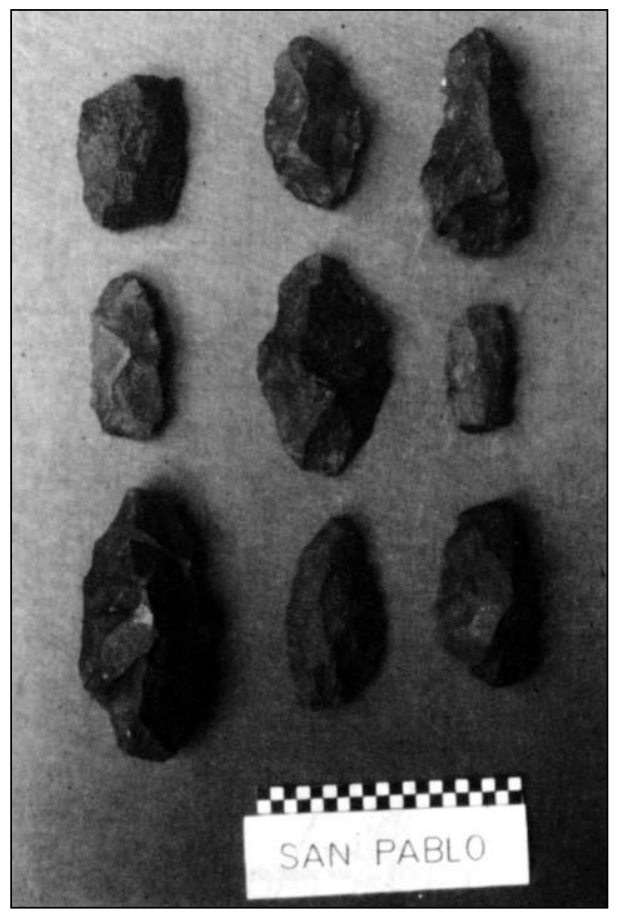

Figura 10. Artefactos líticos de San Pablo de Lípez, algunos en la última etapa de elaboración de puntas de proyectil (basalto negro).

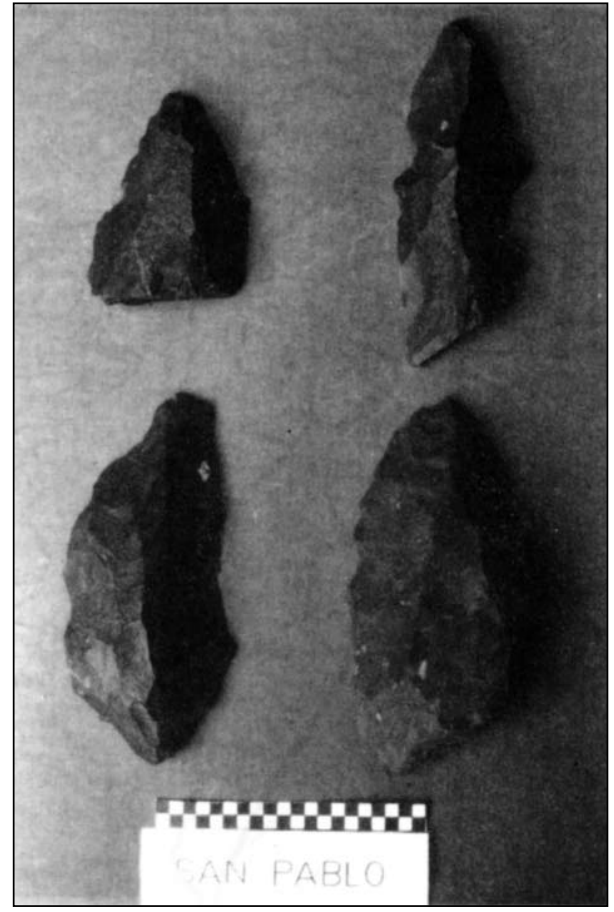

Figura 9. Artefactos líticos de San Pablo de Lípez, elaborados en basalto negro.

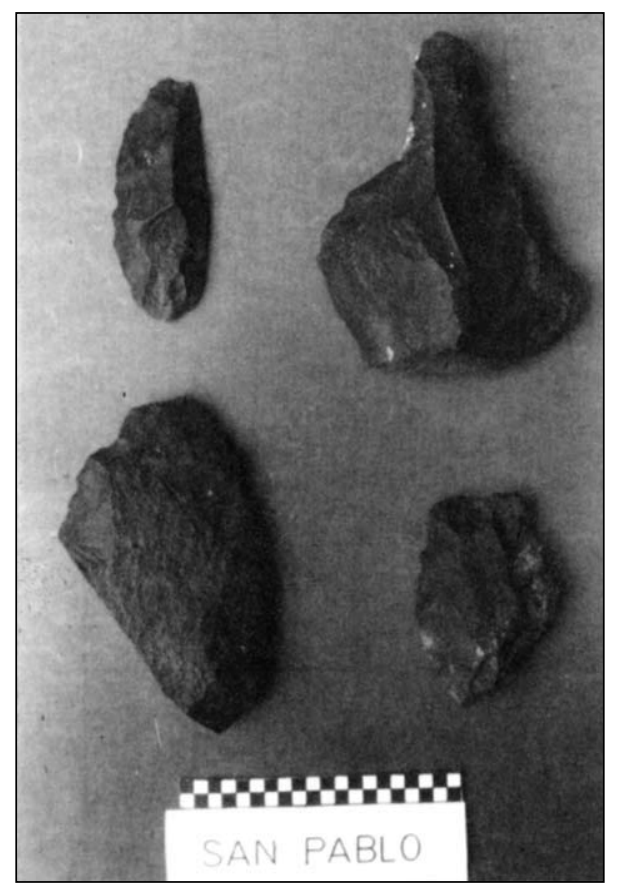

Figura 11. Artefactos líticos de San Pablo de Lípez; entre ellos se puede observar un artefacto que posiblemente se utilizó como pico (basalto negro) 


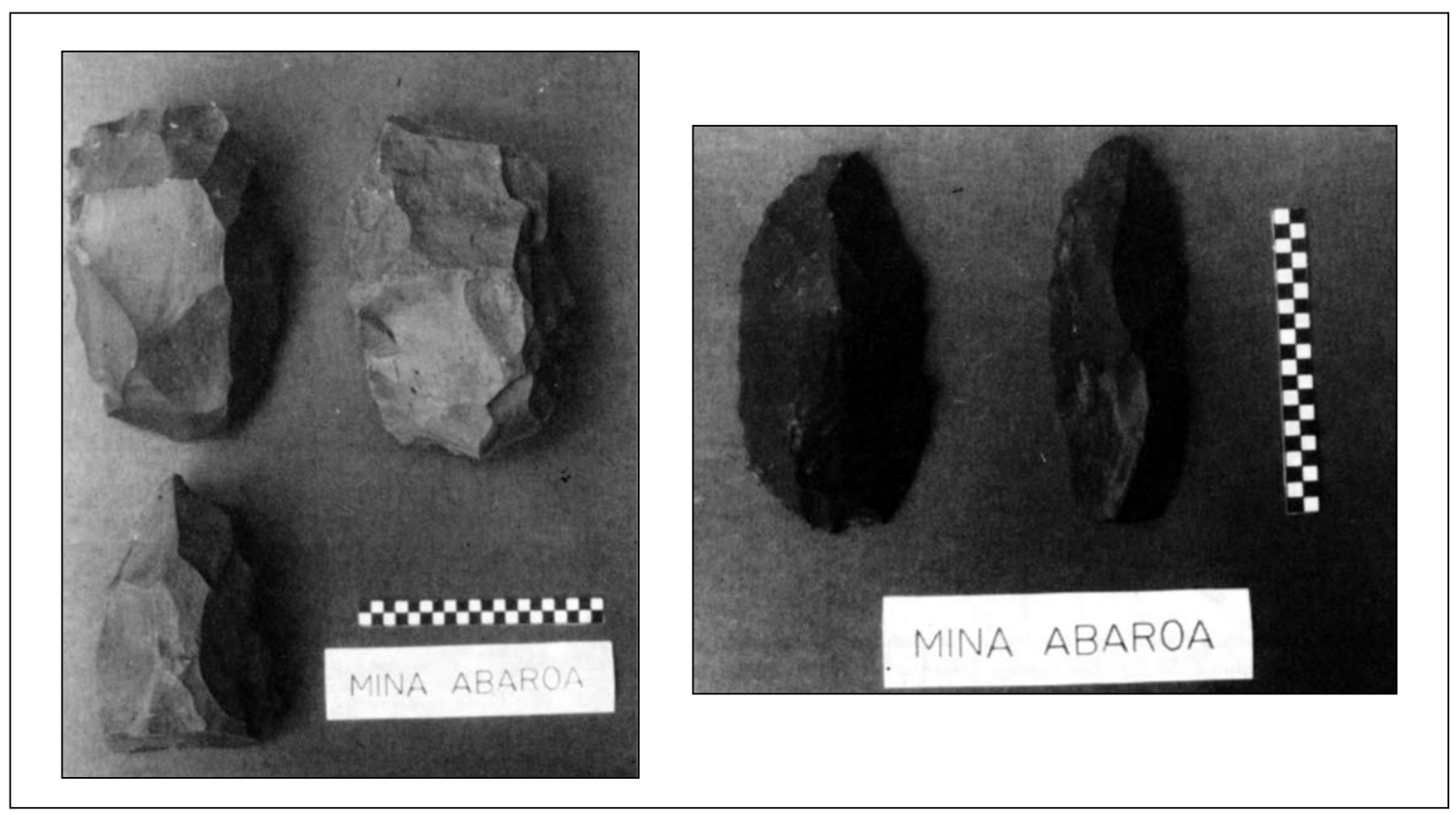

Figura 12. Artefactos líticos de mina Abaroa. Nótense sus grandes dimensiones (obsidiana devitrificada de color verde).

en las terrazas inferiores de los principales ríos de la región, relacionadas directamente con la planicie y que apenas tienen como término medio $2 \mathrm{~m}$ de altura sobre el lecho de los ríos.

Los sitios correspondientes a esta categoría se agrupan principalmente a lo largo de los ríos Quetena y Lípez, y fueron denominados según se van ubicando aguas arriba como I, II, III y IV.

Cada uno de los sitios tiene una estrecha relación entre sí en cuanto a una tipología de artefactos (especialmente puntas de proyectil) y de materia prima compuesta por basalto negro, obsidiana verdosa devitrificada, ópalo y cuarzo lechoso. Por ejemplo, las puntas lanceoladas grandes (largo promedio $70 \mathrm{~mm}$ ) se encuentran en los sitios río Quetena I, II, IV y río Lípez. Puntas triangulares grandes (largo promedio $40.7 \mathrm{~mm}$ ) en río Quetena I y río Lípez I. También se tienen puntas pequeñas: lanceoladas sólo en río Lípez y triangulares en río Quetena II, IV y río Lípez. Estas puntas pequeñas tienen como largo promedio $30 \mathrm{~mm}$, y su característica común es una escotadura central. La talla fue realizada, en todos los casos, a presión; excepcionalmente se tienen puntas con retoque bifacial a presión. Como se podrá observar, es imposible efectuar una diferenciación de tradición cronológica por la diversidad tipológica y por tratarse de sitios superficiales. De manera que no se puede hacer por el momento una relación con yacimientos tradicionales e investigados estratigráficamente. Finalmente, de manera particular trataremos el sitio de Ichu Pampa, al que consideramos como un lugar de caza por tener una variada presencia de artefactos superficiales, de una diversidad de tradiciones, lo que indicaría una sucesión en cuanto a ocupación por distintos grupos humanos en un espacio de tiempo también considerable. El material está distribuido superficialmente en una extensión aproximada de $1500 \mathrm{~m}^{2}$ sobre una terraza de $1.5 \mathrm{~m}$ de altura sobre el lecho del río Lípez. Los instrumentos comprenden desde puntas de proyectil de diversos tipos a raspadores laterales, microrraspadores terminales, microrraspadores circulares, raspadores-perforadores, perforadores, lascas con retoques, etc. No obstante, en el sitio no existe la materia prima utilizada para la fabricación de estos instrumentos, y que consiste en basalto negro-grisáceo, obsidiana verdosa devitrificada, ópalo de diversas tonalidades y cuarzo lechoso. En cuanto a una tipología de las puntas de proyectil, se observa una relación de varios de los tipos presentes en Ichu Pampa con aquellos de los ríos Quetena y Lípez.

Consideraciones acerca de correlaciones inferidas con distintos yacimientos arqueológicos del Paleoindio fueron señaladas en una oportunidad anterior (Berberian y Arellano 1978: 13), las que, sin embargo, 
deben nuevamente ser sujetas a análisis, luego de efectuar excavaciones en varios sitios favorables, ubicados en esta primera etapa de investigación del Paleoindio en Bolivia.

Agradecimientos Hago extensivo mi agradecimiento a los Dres. Clifford Evans y Betty Meggers, por hacer posible mi participación en el Seminario sobre Tecnología del Paleoindio. De la misma forma al Dr. Dennis Stanford y demás participantes, por las enseñanzas impartidas, las que me servirán en el futuro para realizar nuevas investigaciones en Bolivia. A cada uno de ellos, agradecimientos especiales.

\section{REFERENCIAS CITADAS}

AHLFELD, F., 1973. Geografía de Bolivia. Editorial Amigos del Libro, La Paz.

AHLFELD, F. y L. BRANISA, 1960. Geología de Bolivia. Instituto Boliviano del Petróleo, Editorial Don Bosco, La Paz.

AHLFELD F. y A. SCHEIDER-SCHERBINA, 1964. Los yacimientos minerales y de hidrocarburo de Bolivia. Boletín 5 Servicio Geológico de Bolivia.

AVILA, W., 1978. Consideraciones sobre el vulcanismo cenozoico en la cordillera Occidental de Bolivia. Boletín del Servicio Geológico de Bolivia II (1): 31-56.

BARFIELD, L., 1961. Recent discoveries in the Atacama Desert and the Bolivian Altiplano. American Antiquity 27 (1): 93-100.

BERBERIAN, E. y J. ARELLANO, 1978. Los cazadores y recolectores tempranos en la región de Lípez (departamento de Potosí). Instituto Nacional de Arqueología, documentos $37 / 78$.

CARRASCO, R., 1978. Vulcanismo en la región sur-occidental de Bolivia. Boletín del Servicio Geológico de Bolivia II (1): $25-30$.

COURTY, G., 1910. La question du prehistorique américain. Bulletin et Memoires de la Société d'Antropologie du Paris VI (1): 189-190.

CHSLATTER, L.S. y M.H. NEDERLOF, 1966. Bosquejo de la geología y paleogeografía de Bolivia. Boletín 8 , Servicio Geológico de Bolivia.
DOBROVOLNY, E., 1962. Geología del valle de La Paz. Boletín 3 , Servicio Geológico de Bolivia, La Paz.

FERNANDEZ, A., 1972. Geomorfología de la provincia Sud Lípez, Sociedad Geológica Bolivian. Boletín 18: 70-73.

GRAF-MEIER, K., 1977. Nuevos datos palinológicos del Cuaternario Alto de Bolivia. Boletín del Servicio Geológico de Bolivia I (1): 1-14.

JORDAN, L. y E. RIVAS, 1973. Mineralogía y petrografía de la región de laguna Colorada (provincia Sud Lípez del departamento de Potosí). Sociedad Geológica Boliviana. Boletín 19: 125-133.

LE PAIGE, G., 1964. El Precerámico en la cordillera atacameña y los cementerios del Período Agroalfarero de San Pedro de Atacama. Anales de la Universidad del Norte 3, Antofagasta.

MUÑOZ REYES, J., 1977. Geografía de Bolivia. Academia Nacional de Ciencias de Bolivia, La Paz.

PONCE SANGINES, C., 1970. La misión científica francesa Créqui-Monfort. Kollasuyo. Revista de Estudios Bolivianos 71: 104-124.

SERVANT, M., 1977. El cuadro estratigráfico del plio-Cuaternario del Altiplano de los Andes tropicales de Bolivia. Revista de Geociencias 1 (1).

WALKER, E. H., 1949. Andean uplift and erosion surfaces near Uncia, Bolivia. American Journal of Science 247 (9): 646-663. 
